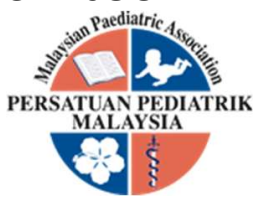

\title{
A-10-YEAR REVIEW ON SURVIVAL RATE AND PROGNOSTIC FACTORS OF EWING FAMILY TUMOUR IN CHILDREN AT HOSPITAL UNIVERSITI SAINS MALAYSIA
}

\author{
Ariffin Nasir, Norhaila Adenam, Surini Yusoff, Fahisham Taib, Norsarwany Mohamad
}

\begin{abstract}
Introduction: Ewing Family Tumour (EFT) is a group of rare malignant and aggressive tumour, with a considerably improved prognosis. However, there is lack of study on the outcome of children with EFT in Malaysia. Objectives: The study aimed to evaluate the Overall Survival (OS) rate, Event Free Survival (EFS) rate and identify the prognostic factors that determined the EFT outcome at Hospital Universiti Sains Malaysia (USM). Methodology: A retrospective record review of children aged 0-18 years with EFT was done. Patients were identified from the registration data in the Oncology Unit and Record Office of Hospital USM. For patients with untraceable information or deceased, a letter was sent to State Registry to obtain the outcome of the patient. The association between demographics and patients' clinical factors was determined using the Cox regression. Survival curves were estimated by the Kaplan-Meier method and were compared using the Log-rank test. Results: There were 51 patients identified but 29 of them were eligible for the study. The mean duration of follow-up was 21 months. The OS rate at 1, 2, 3 and 5 years were $62.1 \%, 44.8 \%, 30.2 \%$ and $21.6 \%$ respectively. The EFS rate at 1, 2, 3 and 5 years were $41.9 \%$, $26.7 \%, 17.8 \%$ and $0 \%$ respectively. Multivariate Cox regression analysis showed that the presence of surgical intervention $(p=0.030)$ and major complications $(p=0.045)$ were the significant prognostic factors to the survival of EFT. Conclusion: The survival rate of EFT among our patients was comparable to other developing countries, with surgical intervention and the presence of major complications as independent prognostic factors.
\end{abstract}

Keywords: Ewing Family Tumour, Overall Survival Rate, Event Free Survival, Children

DOI: $10.51407 / \mathrm{mjpch} . v 27 \mathrm{i} 2.138$

\section{Introduction}

Ewing Family Tumour (EFT) is a malignant small, round, blue cell tumour, described as the "diffuse endothelioma of bone"[1], including Askin and Primitive Neuroectodermal Tumour (PNET). The incidence of EFT varies among the different racial groups, ages and is much rarely seen in Asian. It has a peak incidence at the age of 15 years and is more common in males compared to females, with the ratio of 1.3 to $1.5: 1$.

The 5-year survival rate for children with bone cancer has improved from 49\% (1975-1984) to $63 \%$ (1985-1994) for both Osteosarcoma and
Received: 22 December 2020; Accepted revised

manuscript: 19 October 2021

Published online: 2 December 2021
Ewing sarcoma [2]. The improved chemotherapy regimens have considerably improved the EFT prognosis [3,4]. The Overall Survival (OS) is defined as the time from the diagnosis until death from any causes or until the last follow-up whereas Event Free Survival (EFS) is the interval

Department of Paediatrics, School of Medical Sciences, Health Campus, Universiti Sains Malaysia, 16150 Kota Bharu, Kelantan, Malaysia

Hospital Universiti Sains Malaysia, 16150 Kota Bharu, Kelantan, Malaysia

Corresponding Author:

Norsarwany Mohamad, Department of Paediatrics, School of Medical Sciences, Health Campus, Universiti Sains Malaysia, 16150 Kota Bharu, Kelantan, Malaysia

Tel.: 6097676540 Email: sarwany@usm.my 
between the date of diagnosis and the earliest occurrence of the following events: induction failure, relapse, death from any cause, last contact and development of a second malignancy [5]. Although there have been studies conducted worldwide, there is no documented comprehensive work to study EFT in the authors' knowledge. This is primarily patients with EFT requiring a dedicated paediatric orthopaedic service to complement the medical approach despite many presented with big tumour size and metastases. Hospital Universiti Sains Malaysia (USM) has provided a multidisciplinary approach to musculoskeletal and bony oncology since 1990's.

The study was conducted to evaluate the OS, EFS rate and identify the prognostic factors of patients with EFT at Hospital USM.

\section{Methods}

\section{Study area and patients}

Hospital USM is a tertiary centre covering Paediatric Oncology services in the East Coast of Peninsular Malaysia. It has 23 beds to care for paediatric oncology cases and mostly cater to children originated from Northeast of Peninsular Malaysia. Some patients may come from other states and refer to our centre following the diagnostic procedure, mainly due to family preferences.

All children aged 0-18-year-old at the time of diagnosis with EFT features confirmed by Histopathology Examination (HPE) findings, fulfilling ICD10-C41.9 criteria and diagnosed from $1^{\text {st }}$ January $2002-31^{\text {st }}$ December 2012, were included. Untraceable case notes, those who were transferred to another hospital for treatment or continuation of chemotherapy were excluded.

\section{Treatment protocols}

Children with EFT diagnosis were treated with either Euro Ewing 99, CAV/IE protocol or Memorial Sloan Kettering (MSK) protocol depending on the type of tumour. The Ifosfamide, Carboplatin and Etoposide (ICE) protocol was the primary chemotherapy regime for palliative chemotherapy.

\section{Study design}

A retrospective record review study was performed using a registration list from paediatric, orthopaedic and record office of Hospital USM. The code number was given to each patient to ensure anonymity. A total of 51 registered cases were identified. However, 22 cases have to be excluded due to incorrect HPE of EFT criteria and missing folders. Therefore, a total of 29 patients were enrolled. The data was collected using a standardized case recording form to capture the demographic data, the disease status, staging, tumour size from CT scan/MRI estimation at diagnosis and the chemotherapy regime for the EFT treatment.

\section{Consent and ethical approval}

Parents of the enrolled patients were approached during their regular follow-up either from the oncology clinic or via phone call. The uncontactable patients were excluded. The study was approved by Human Research and Ethical Committee (HREC) USM [USM/JEPeM/ 278.5. (3.3)]. Information regarding the date and cause of death of the patients were obtained from State Registry.

\section{Outcomes measures}

The outcome of the study was to obtain the EFS and OS for EFT. The prognostic factors identified affecting the OS and EFS were explored.

\section{Statistical analysis}

The statistical analysis was performed using SPSS version 19. Descriptive data were analysed where the numerical data were expressed in mean (SD) or number and percentage (n, \%). EFS and OS were calculated using the Kaplan-Meier method. A log-rank test was used to compare the survival curves of the subgroups. Univariate Cox proportional hazard model was used to explore predictors. These variables include gender, age at diagnosis, size of tumour, pelvic versus non-pelvic site of tumour, lung metastasis, skip bone metastasis, disease profile, chemotherapy regime, surgical intervention and complications. The factors with P-value less than 0.25 were included in the multivariate Cox proportional hazard. A Pvalue of $<0.05$ was considered significant. 


\section{Results}

There were 51 children aged 0-18 years who were diagnosed with Ewing Family Tumour (EFT) from $1^{\text {st }}$ January 2002 to $31^{\text {st }}$ December 2012. Only 29 patients were eligible for the study. The excluded cases were Chronic Osteomyelitis $(n=6)$, Osteosarcoma $\quad(n=5), \quad$ Langerhans Cell Histiocytosis $(n=1)$, normal HPE result $(n=2)$, missed follow up $(n=1)$ and unavailable case notes $(n=5)$. Two of the confirmed EFT cases were also excluded because one patient was managed in the other hospital and another one was diagnosed outside the study period.

\section{Demographic profile of children with Ewing Family Tumour}

Table 1 showed the demographic profile of children with EFT at HUSM from 2002-2012. The minimum age of the patient was 4.6 years and the oldest age was 17.7 years. The mean age was 12.0 years old (SD: 3.74). The majority of patients were older than 10 years-of-age (79.3\%). Male comprised of $55.2 \%$ of the cases and female was $44.8 \%$, with $86.2 \%$ were Malay ethnicity.

The majority of patients were histologically diagnosed as Peripheral Neuroectodermal Tumour (PNET) (55.2\%) followed by classical Ewing sarcoma (37.9\%) and extra-osseous group (6.9\%). No Askin tumour and atypical subtype EFT were documented. A large proportion of tumour (72.4\%) came from the non-pelvic site which includes extremities (55.2\%) and axial (17.2\%). The tumour size ranges from 4 to $30 \mathrm{~cm}$ (mean SD: 11.5). The majority of the children have tumour sizes between $10-15 \mathrm{~cm}$. Only 3 patients $(10.3 \%)$ had tumour less than $8 \mathrm{~cm}$ in diameter. Fifteen patients (51.7\%) have localized disease and 11 (37.9\%) have evidence of metastasis at the time of diagnosis. Among those with metastasized diseases, 11 (37.9\%) were documented to have the tumour in the lung and $3(10.3 \%)$ had skip bone metastasis. The majority of our patients (16 children) did not undergo any surgical intervention. Of those with surgical intervention, 13 of them had a wide tumour resection and only 1 patient had partial surgical resection due to inaccessible tumour.

More than half of patients $(15,51.7 \%)$ did not develop major complications. Documented sequalae were significant sepsis $(8,26.7 \%)$ and neurology complications such as encephalitis (3,
10\%). Other documented complications were severe pneumonia $(n=1)$, deep vein thrombosis (DVT) $(n=1)$ and liver toxicity grade III $(n=1)$. Many of our patients defaulted to treatment either in the initial phase, during the treatment or at the end of the treatment (76.7\%). Only 7 (23.3\%) patients complied to follow up. At the endpoint of the study period, $79.3 \%(n=23)$ patients were deceased, while $17.2 \%(n=5)$ patients were still alive. 
Table 1. Demographic profile and tumour characteristics of children with EFT $(n=29)$

\begin{tabular}{|c|c|c|}
\hline Features & $\mathrm{n}$ & $\%$ \\
\hline \multicolumn{3}{|l|}{ Gender } \\
\hline Male & 16 & 55.2 \\
\hline Female & 13 & 44.8 \\
\hline \multicolumn{3}{|l|}{ Age } \\
\hline$<10$ years & 6 & 20.7 \\
\hline$\geq 10-18$ years & 23 & 79.3 \\
\hline \multicolumn{3}{|l|}{ Race } \\
\hline Malay & 25 & 86.2 \\
\hline Chinese & 1 & 3.5 \\
\hline Indian & 3 & 10.3 \\
\hline \multicolumn{3}{|l|}{ Primary Tumour site } \\
\hline Extremities & 16 & 55.2 \\
\hline Pelvis & 8 & 27.6 \\
\hline Axial & 5 & 17.2 \\
\hline \multicolumn{3}{|l|}{ Disease Profile } \\
\hline Classical Ewing Sarcoma & 11 & 37.9 \\
\hline $\mathrm{PNET}^{1}$ & 16 & 55.2 \\
\hline Extraosseous & 2 & 6.9 \\
\hline \multicolumn{3}{|l|}{ Size of tumour } \\
\hline$<8 \mathrm{~cm}$ & 3 & 10.3 \\
\hline$\geq 8 \mathrm{~cm}$ & 21 & 72.4 \\
\hline Undocumented & 5 & 17.3 \\
\hline \multicolumn{3}{|l|}{ Metastasis at diagnosis } \\
\hline Yes & 11 & 37.9 \\
\hline No & 15 & 51.8 \\
\hline Undocumented & 3 & 10.3 \\
\hline \multicolumn{3}{|l|}{ Skip bone metastasis at diagnosis } \\
\hline Yes & 3 & 10.3 \\
\hline No & 16 & 55.2 \\
\hline Undocumented & 10 & 34.5 \\
\hline \multicolumn{3}{|l|}{ Surgical intervention } \\
\hline Surgery & 13 & 44.8 \\
\hline No surgery & 16 & 55.2 \\
\hline \multicolumn{3}{|l|}{ Complications } \\
\hline Sepsis & 8 & 27.6 \\
\hline Neurology complication & 3 & 10.3 \\
\hline Others ${ }^{4}$ & 3 & 10.3 \\
\hline Nil & 15 & 51.8 \\
\hline \multicolumn{3}{|l|}{ Default to follow up and treatment } \\
\hline Yes & 23 & 79.3 \\
\hline No & 6 & 20.7 \\
\hline \multicolumn{3}{|c|}{ Final outcome at the end of study period } \\
\hline Alive & 5 & 17.2 \\
\hline Dead & 23 & 79.4 \\
\hline Missing data & 1 & 3.4 \\
\hline
\end{tabular}




\section{Survival analysis}

The overall median survival time was 18 months (95\% Cl: 12.7, 23.3). The Overall Survival (OS) rate was $75.9 \%$ at 6 months, $62.1 \%$ at 1 year, $44.8 \%$ at 2 years, $30.2 \%$ at 3 years and $21.6 \%$ at 5 years after diagnosis.
The mean of EFS was 23.2 months $(95 \% \mathrm{Cl}: 11.5$, 35). The EFS rate was $64.8 \%$ at 6 months after diagnosis, $41.9 \%$ at 1 year, came down to $26.7 \%$ and $17.8 \%$ at 2 years and 3 years respectively. At 5 years, the EFS rate was $0 \%$ (Figure 1).
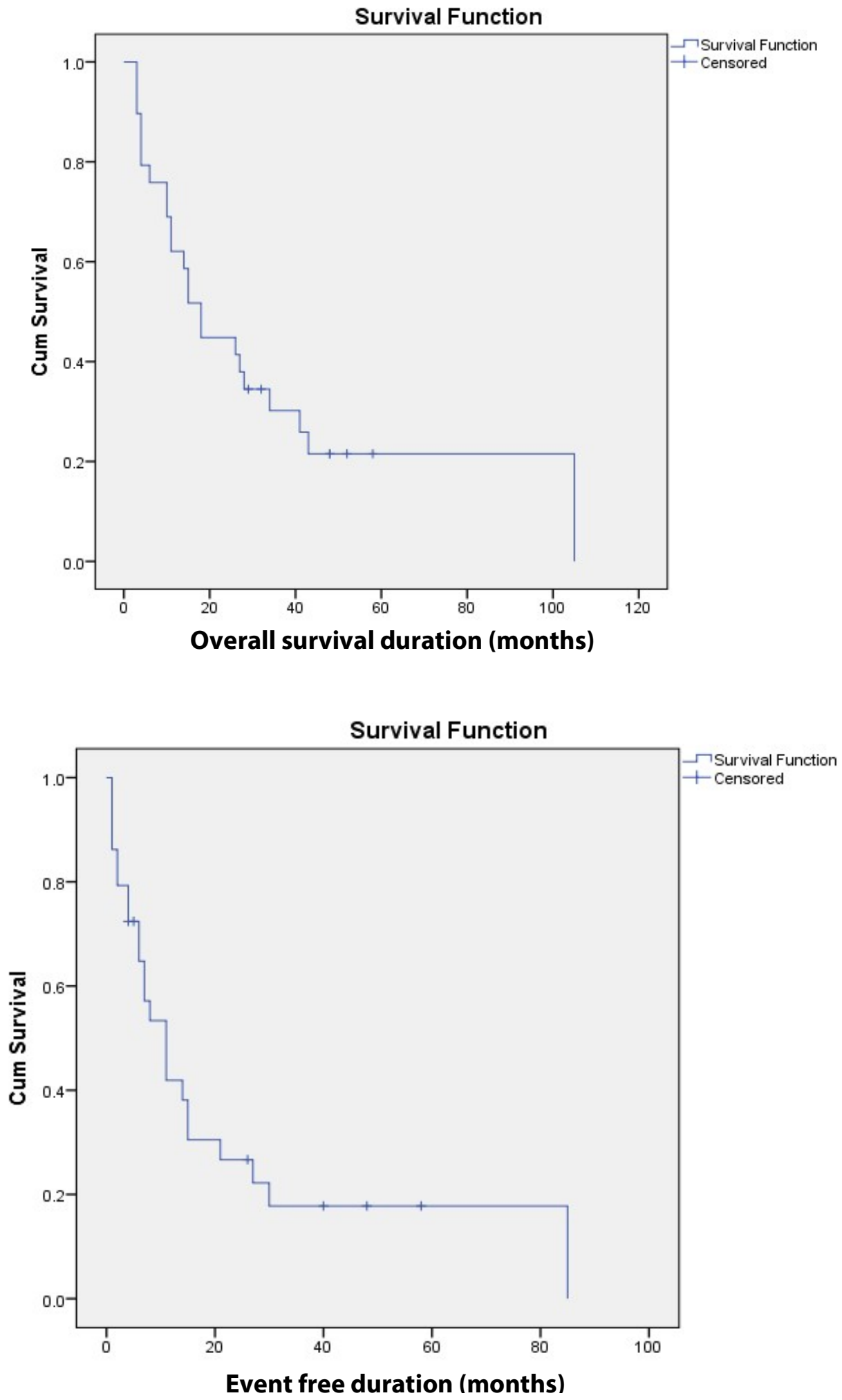

Figure 1. Kaplan-Meier survival estimation of OS and EFS for children with EFT at HUSM 
There were five potential prognostic factors for poor prognosis in EFT which include pelvis versus non-pelvis tumour site, skip bony metastasis at diagnosis, type of chemotherapy regime, the presence of complications and the surgical intervention. These variables were subjected to multivariate analysis (Table 2).

Table 2. Simple Cox Regression analysis of prognostic factors for survival of children with EFT

\begin{tabular}{|c|c|c|c|c|}
\hline Variables & $\begin{array}{l}\text { Regression } \\
\text { Coefficient }\end{array}$ & $\begin{array}{l}\text { Crude Hazard Ratio } \\
(95 \% \mathrm{Cl})\end{array}$ & $\begin{array}{l}\text { Wald } \\
\text { statistic }\end{array}$ & p-value \\
\hline \multicolumn{5}{|l|}{ Age } \\
\hline$<10$ years & 0 & 1.0 & & \\
\hline$\geq 10$ - 18 years & -0.28 & $0.76(0.28,2.06)$ & 0.30 & 0.58 \\
\hline \multicolumn{5}{|l|}{ Gender } \\
\hline Male & 0 & 1.0 & & \\
\hline Female & 0.027 & $1.03(0.44,2.39)$ & 0.04 & 0.95 \\
\hline \multicolumn{5}{|l|}{ Ethnicity } \\
\hline Non-Malay & 0 & 1.0 & & \\
\hline Malay & 0.29 & $1.34(0.39,4.54)$ & 0.22 & 0.64 \\
\hline \multicolumn{5}{|l|}{ Tumour size } \\
\hline$<8 \mathrm{~cm}$ & 0 & 1.0 & & \\
\hline$\geq 8 \mathrm{~cm}$ & 0.99 & $2.71(0.36,20.4)$ & 0.93 & 0.33 \\
\hline \multicolumn{5}{|l|}{ Site of tumour } \\
\hline Non pelvic & 0 & 1.0 & & \\
\hline Pelvic & 0.68 & $1.96(0.78,4.92)$ & 2.07 & 0.15 \\
\hline \multicolumn{5}{|l|}{$\begin{array}{l}\text { Metastasis } \\
\text { at diagnosis }\end{array}$} \\
\hline No & 0 & 1.0 & & \\
\hline Yes & 0.46 & $1.59(0.4,2.61)$ & 0.96 & 0.33 \\
\hline \multicolumn{5}{|c|}{$\begin{array}{l}\text { Skip Metastasis of bone } \\
\text { at diagnosis }\end{array}$} \\
\hline No & 0 & 1.0 & & \\
\hline Yes & 1.27 & $3.56(0.83,15.2)$ & 2.93 & 0.087 \\
\hline \multicolumn{5}{|l|}{ Major } \\
\hline Complications ${ }^{1}$ & 0 & 1.0 & & \\
\hline No & 0.80 & $2.20(0.95,5.23)$ & 3.40 & 0.065 \\
\hline Yes & & & & \\
\hline \multicolumn{5}{|c|}{ Surgical intervention } \\
\hline No surgery & 0 & 1.0 & & \\
\hline Surgery & -0.91 & $0.40(0.16,0.10)$ & 3.88 & 0.049 \\
\hline
\end{tabular}

${ }^{1}$ Major Complications (Cardiac, neurology, coagulopathy and bleeding, sepsis, complication of treatment (DVT, Liver toxicity), secondary malignancy) 


\section{Multiple Cox Regression Analysis for EFT}

All five potential prognostic factors were used as a preliminary model and proceeded with logistic Regression analysis. Only two factors, which were major complications and surgical intervention, were identified to have statistically significant value. The significant variables were checked for interaction and were found with statistically no interaction ( $p$-value 0.72) between them. Both factors were included in the final model. Hazard of dying is increased by 4 times when there were major complications, $(b=1.41, \quad H R=4.1 \quad(95 \%$ $\mathrm{Cl}: 1.03,16.2 ; \mathrm{p}=0.045)$. The hazard of dying is decreased by $80 \%$ when patients went for surgical intervention, $(b=-1.54, H R=0.21(95 \% C l$; $0.53,0.86 ; p=0.030)$. Therefore, surgical intervention is a protective factor to EFT survival (Table 3).

Table 3. Multiple Cox Regression analysis of prognostic factors for survival in children with EFT.

\begin{tabular}{ccccc}
\hline Variables & $\begin{array}{l}\text { Regression } \\
\text { Coefficient(b) }\end{array}$ & $\begin{array}{l}\text { Adjusted Hazard Ratio } \\
(\mathbf{9 5 \%} \mathbf{C l})\end{array}$ & $\begin{array}{l}\text { Wald } \\
\text { statistic }\end{array}$ & p-value \\
\hline $\begin{array}{c}\text { Major complications } \\
\text { No }\end{array}$ & 0 & 1 & & 0.045 \\
Yes & 1.41 & $4.10(1.03,16.2)$ & 4.03 & 0.030 \\
Surgical intervention & 0 & 1 & 4.69 & $0.86)$ \\
No surgery & -1.54 & $0.21(0.53,0.86)$ & & \\
Surgery & & &
\end{tabular}

'Major complications: Neurology complication, cardiac complication, severe infection and sepsis, coagulopathy and bleeding, secondary malignancy, complication of treatments (DVT, Liver toxicity, etc).

\section{Discussion}

EFT is a very rare malignant disease. We were only able to enroll 29 patients who fulfilled the criteria for the study. EFT is more common in males as compared to females, with an incidence of 1.3 times higher in our study. This was comparable to the worldwide ratio where documented incidence was between 1.3 and 1.5 higher in males compared to females [6]. The majority of the patients were Malay (86.2\%), followed by Indian (10.3\%) and Chinese (3.4\%) ethnic groups. The East Coast of Peninsular Malaysia, especially Kelantan, was homogenously inhabited by Malay ethnicity.

Data taken from the Surveillance, Epidemiology and End Result (SEER) program in the USA, between the year of 1973-1987, found 420 patients of Ewing Sarcoma [7]. The number of the sample in our study was relatively small due to the rarity of EFT disease especially among the Asian population. The majority of the parents for our patients came from a low educational background whereby $24.1 \%$ had completed primary and secondary education. These may explain the higher number of defaulted treatments $(79.3 \%)$ and it was noted that $34.5 \%$ of our patients had sought for alternative therapies. Cultural influence and financial constraint have led to a poor understanding of the treatment for EFT. The mean age was 12 years old (SD: 3.74). The majority were in the older subgroup, age $\geq 10$ years $(79.3 \%)$. There was no infant in our study with only 1 patient who was under 5 , which was slightly lower compared to previous study [8]. The incidence was higher in older age group, with $20.7 \%$ of patients were less than 10 years old and $79.3 \%$ were more than 10 years old. This incidence was similar to Stiller et al [6].

The most common primary tumour site was similar with previous studies, with non-pelvic site (72.4\%) coming from extremities (55.2\%) and axial (17.2\%), and a majority of those were femur in origin. PNET (55.2\%) was the commonest EFT. Generally, the classical Ewing Sarcoma was the commonest and followed by PNET [8]. Extraosseous Ewing sarcoma involving the lungs as primary tumour site $(3.4 \%)$ and femur (3.4\%) was seen in 2 patients. These subtypes are rare, and the exact incidence could not be ascertained [9].

Twenty-one patients (72.4\%) possessed tumour size of $\geq 8 \mathrm{~cm}$, at the time of presentation. 
Previous studies documented a much lower tumour size compared to our findings $[8,10,11]$. The cut off $8 \mathrm{~cm}$ of tumour size was based on American Joint Committee on Cancer staging [12]. This result could reflect the nature of aggressiveness, rapidly progressive disease and late presentation as the contributing factors. EFT is known to be a highly malignant tumour with the incidence of metastasis around 20-50\% $[8,10,11]$. Among those with distant metastasis, $31-51 \%$ was found in the lungs and $25-42 \%$ in the bone $[6,9]$. Our study showed a comparable finding with $37.9 \%$ of patients who had metastasis to the lungs and $10.3 \%$ with skip bone metastasis.

More than half of patients (55.2\%) did not undergo surgical intervention. Complete resection was performed in $41.4 \%(n=12)$ and one patient $(3.4 \%)$ needed partial resection due to inaccessible lesion (vertebra lumbar lesions). The majority of patients (92\%) with EFT in other centres would undergo surgical intervention $[10,13]$. There were multifactorial reasons for the lack surgical of intervention in our study such as cultural and family influences, late presentation with multiple distant metastasis and default on follow-up. The protocols used for treatment were Ewing Euro 99 (27.6\%), PNET (10.3\%) and Memorial Sloan Kettering (MSK) (6.9\%). Those who were above 12 years old received $\mathrm{CAV} / \mathrm{IE}$ regime protocol (48.3\%).

There were significant findings such as defaulted to follow up and treatment at $79.3 \%$. At the end of 10 years study period, $79.3 \%$ of patients were deceased, with 5 patients (17.2\%) were alive. The mortality in our population is higher compared to other studies $[10,14]$.

\section{Overall Survival of EFT}

In our study, OS for EFT was $62.1 \%$ at 1 year, $44.8 \%$ at 2 years, $30.2 \%$ at 3 years, $21.6 \%$ at 5 years and $0 \%$ at 10 years. This was an almost comparable result with the Taiwanese where their OS rate were $45.5 \%$ at 2 years and $22.7 \%$ at 3 years [6]. In the US, the 5 years OS rate was up to $63.5 \%$ [10] and others reported as high as $50-70 \%$ [13].

\section{Event Free Survival of EFT}

We found that the median survival time for EFT was 11 months (95\% Cl:6.1, 15.9). Overall EFS at 1, 2,3 and 5 years were $26.7 \%, 26.7 \%, 17.8 \%$ and $0 \%$ respectively. The EFS in our context is considered poor compared to other developing countries. The 5 and 10 years EFS rates were 21\% and 19\% respectively in Turkey [8]. The 5 years EFS was higher in developed nations ranging from 41$55.1 \%[13,14]$.

\section{Treatment outcomes according to risk factors} Younger age was found to be better prognostically with additional localised disease and non-pelvic tumour [14] with OS rate $88.1 \%$ [13]. Others also demonstrated that younger age ( $<15$ years vs $\geq 15$ years) had significant influences on the EFS [15]. The contrasting finding in our study where the older age group survived better and factors that influenced the outcome was the small tumour size, younger age group and disease status either localized or metastasized.

Generally, non-Malay has a better survival compared to the Malay ethnic group, however, this was not statistically significant $(p=0.179)$. The simple cox regression analysis showed HR of 1.34 $(95 \% \mathrm{Cl} ; 0.39,4.54)$ among Malay race to have the poorer outcome $(p=0.64)$. Simple cox regression model showed female patient has a higher risk for poor outcome compared to male $(p=0.950)$. HR female compared to male of $1.03(95 \% \mathrm{Cl} ; 0.44$, 2.39). However, in the previous study, male patients were recorded to have poorer survival outcomes with the risk of dying 1.3 times to females $(p=0.03$ ) [16]. Most studies did not show any significant gender differentiation towards survival.

The site of the tumour plays a significant prognostic factor. In our study, the OS $(p=0.133)$ and EFS $(p=0.396)$ of the tumour from non- pelvic were not superior to the tumour at pelvic area. The pelvic site was found to have a poorer survival rate compared to the non-pelvic site, but this was statistically insignificant. The pelvic site of tumour is known as one of the poor prognostic factors in previous study with 5 years OS (13\%) as compared to the non-pelvic site $(37 \%)[8,14]$. Rodriguez-Galindo et al. identified patients with pelvic tumour site, age $\geq 14$ years old and localized disease as an intermediate-risk group with 5 years OS of $53.8 \%$ compared to $64.9 \%$ of the favorable risk group (age $<14$ years with localized, non-pelvic tumours) with a p-value 0.001 [13].

Tumour size more than $8 \mathrm{~cm}$ was an independent prognostic to poor survival rate $[8,13]$. A previous study in the adult population found that there 
was no significant difference in prognostic factors in the adult compared to children [17]. Lung metastasis was more common site of metastasis than skip bone metastasis in our study. Univariate analysis of lung metastasis was not statistically significant as prognostic factors with $\mathrm{HR}$ of 1.59 $(95 \% \mathrm{Cl} ; 0.63,4.03)$ and $\mathrm{p}$-value of 0.33 but the skip bone metastasis was a potential prognostic factor (HR 3.56, 95\% Cl: 0.83, 15.2), $\mathrm{p}=0.087$.

Multivariate analysis again showed that surgical intervention and major complications were the independent prognostic factors with HR of 0.21 $(95 \% \mathrm{Cl} ; 0.53,0.86 ; \mathrm{p}=.03)$ and $4.1(95 \% \mathrm{Cl} ; 1.03$, $16.2 ; p=0.045)$ respectively.

\section{Limitations}

This study has few limitations. The sample size was too small due to the rarity of the disease. Searching for the retrospective record information was challenging due to poor documentation. The enrolled patients were managed by two different teams; primarily because of age differences hence leading to different treatment plans and protocols. There was also issue related to missing case notes which influence the total sample size number.

\section{Conclusion}

Most patients in our study were males, with the mean age were 12 years old, commonest site of presentation was from the extremities and had evidence of metastasis at the time of diagnosis. Late presentation (large tumour bulk), defaulted to treatment and non-surgical interventional approach have largely affected the study outcome. The unfavorable presence of distant metastasis at the time of diagnosis has affected the prognostication in our study. The absence of major complications and surgical intervention was the identified independent prognostic factors for survival in EFT patients in our population.

\section{References}

[1] Ewing J. Classic in oncology. Diffuse endothelioma of bone. Cancer Journal of Clinician. 1972; 22(2): 95-8.
[2] National Cancer Institute. Physician Data Query (PDQ); Ewing Sarcoma Treatment. Journal of the National Cancer Institute. 2013.

[3] Burgert EO, Jr, Nesbit ME, Garnsey LA, Gehan EA, Herrmann J, Vietti TJ, et al. Multimodal therapy for the management of non-pelvic, localized Ewing's sarcoma of bone: intergroup study of IESS-II. Journal of Clinical Oncology. 1990; 8:1514-24.

[4] Grier HE, Krailo M.D, Tarbell NJ, Link MP, Fryer CJ, Pritchard DJ. Addition of ifosfamide and etoposide to standard chemotherapy for Ewing's sarcoma and primitive neuroectodermal tumor of bone. $\mathrm{N}$ Engl J Med. 2003; 348: 694-701.

[5] Tang AP. Bentzen SM Chen EX, Siu LL. Surrogate end points for median overall survival in metastatic colorectal cancer: literature-based analysis from 39 randomized controlled trials of first line chemotherapy. J of Clin Oncology. 2007; 25(29): 4562-8.

[6] Stiller CA, Parkin DM. Geographic and ethnic variation in the incidence of childhood cancer. British Medical Bulletin. 1996; 52(4): 682-708.

[7] American Cancer Society. Cancer Fact and Figures 2016. Altanta: American Cancer Society. 2016.

[8] Kutluk MT, Yalcin, B, Akyuz CA, Varan A. Treatment result and prognostic factors in Ewing Sarcoma. Paediatric Hematology and Oncology. 2004; 21:597-610.

[9] Van den Berg $\mathrm{H}$, Heinen RC, Vander Pal HJ, Merks JH. Extra-osseous Ewing sarcoma. Paeditric Hematology and Oncology. 2009; 26(4): 175-85.

[10] Kai LH, Cheng FC, Po KW, Paul CHC, Wei MC, Chien LL, Tan HC. Clinical outcomes and prognostic factors of Ewing Sarcoma: A clinical analysis of 12 patients in Taiwan. Journal of the Chinese Medical Association. 2012; 75(1): 16-20.

[11] Rodriguez-Galindo C, Liu T, Krasin MJ, Wu J, Billups CA, Daw NC, et al. Review of St Jude Children's Research Hospital Studies: Analysis of prognostic factors in EFTS. American Cancer Society. 2007; 110(2): 375-84. 
[12] American Joint Committee on Cancer Bone. AJCC Cancer Staging Manual $7^{\text {th }}$ edition. New York: Springer-Verley. 2010. p. 281-7.

[13] Bacci G, Longhi A, Ferrari S, Versari M, Bertoni F. Prognostic factors in non-metastatic Ewing's Sarcoma tumor of bone. An analysis of 579 patients treated in a single institution with adjuvant or neoadjuvant chemotherapy between 1972 and 1998. Acta Oncologica. 2006; 45: 469-75.

[14] Rodriguez-Galindo, C., Spunt, S.L., Pappo, A.S. Treatment of Ewing Sarcoma family of tumors: current status and outlook for the future. Med Pediatr Oncology. 2003; 40:27687.

[15] Yonemari K, Yamaguchi U, Kaneko M, Uno H, Takeuchi $M$, Ando $M$, et al. Prediction of response and prognostic factor of Ewing Family Tumor in a low incidence population. Journal Cancer Research and Clinical Oncology. 2008; 134(3): 389-95.

[16] Cotterill SJ, Ahrens S, Paulussen M, Jurgens HF, Voute PA, Gadner H, Craft AW. Prognostic factors in Ewing's Tumor of bone: Analysis of 975 patients from the European Intergroup Cooperative Ewing's Sarcoma Study Group. Journal of Clinical Oncology. 2000; 18(17): 3108-14.

[17] Bacci G, Ferrari S, Bertoni F, Rimondini S, Longhi $A$, Bacchini $P$, et al. Prognostic factors in non-metastatic Ewing's Sarcoma of bone treated with adjuvant chemotherapy: Analysis of 359 patients at the Istituto Ortopedico Rizzoli. Journal of Clinical Oncology. 2000; 18(1): 4. 